\title{
Accuracy of nonmolecular identification of growth-hormone- transgenic coho salmon after simulated escape
}

\author{
L. F. Sundström, ${ }^{1}$ M. Lõhmus, ${ }^{2}$ and R. H. Devlin ${ }^{3}$ \\ DFO Centre for Aquaculture and Environmental Research, 4160 Marine Drive, West Vancouver, British Columbia V7V 1 N6 Canada
}

\begin{abstract}
Concerns with transgenic animals include the potential ecological risks associated with release or escape to the natural environment, and a critical requirement for assessment of ecological effects is the ability to distinguish transgenic animals from wild type. Here, we explore geometric morphometrics (GeoM) and human expertise to distinguish growth-hormone-transgenic coho salmon (Oncorhynchus kisutch) specimens from wild type. First, we simulated an escape of 3-month-old hatchery-reared wild-type and transgenic fish to an artificial stream, and recaptured them at the time of seaward migration at an age of 13 months. Second, we reared fish in the stream from first-feeding fry until an age of 13 months, thereby simulating fish arising from a successful spawn in the wild of an escaped hatcheryreared transgenic fish. All fish were then assessed from photographs by visual identification (VID) by local staff and by GeoM based on 13 morphological landmarks. A leave-one-out discriminant analysis of GeoM data had on average $86 \%$ ( $72-100 \%$ for individual groups) accuracy in assigning the correct genotypes, whereas the human experts were correct, on average, in only $49 \%$ of cases (range of $18-100 \%$ for individual fish groups). However, serious errors (i.e., classifying transgenic specimens as wild type) occurred for $7 \%$ (GeoM) and $67 \%$ (VID) of transgenic fish, and all of these incorrect assignments arose with fish reared in the stream from the first-feeding stage. The results show that we presently lack the skills of visually distinguishing transgenic coho salmon from wild type with a high level of accuracy, but that further development of GeoM methods could be useful in identifying second-generation fish from nature as a nonmolecular approach.
\end{abstract}

Key words: aquaculture; coho salmon (Oncorhynchus kisutch); ecological risk; genetic modification; invasive species; morphology analysis.

\section{INTRODUCTION}

Invasive species can cause large ecological impacts on receiving ecosystems and cause great economic costs (Lovell et al. 2006, Reaser et al. 2007). More recently, genetic modification of a large number of organisms by transgenesis raises concern that similar consequences may arise should such organisms escape or be released into natural ecosystems (Tiedje et al. 1989, Snow et al. 2005). In some cases, transgenic organisms may pose little threat to natural populations because they are often modified for traits that are likely to confer a survival disadvantage under natural conditions. However, some transgenic animals may pose a greater threat than alien species when the transgenic animals share similar evolutionary history with local populations.

Manuscript received 7 October 2014; accepted 10 December 2014. Corresponding Editor: M. E. Hellberg.

${ }^{1}$ Present address: Uppsala University, Evolutionary Biology Centre, Department of Ecology and Genetics/Animal Ecology, Norbyvägen 18D, SE-75236 Uppsala, Sweden.

${ }^{2}$ Present address: Karolinska Institute, Institute for Environmental Medicine, Nobels väg 13, SE-17177 Stockholm, Sweden.

${ }^{3}$ Corresponding author.

E-mail: robert.devlin@dfo-mpo.gc.ca
A range of containment strategies (physical, geographical, and biological) has been proposed to reduce the risk of transgenes entering the wild or causing harm should escapes occur (Devlin and Donaldson 1992, Devlin et al. 2006, Kapuscinski et al. 2007, Mair et al. 2007). However, methods explored to date have not provided complete containment as would be expected for commercial scales (Devlin et al. 2010). To minimize ecological harm from escaped transgenic animals, it would be beneficial to be able to quickly identify transgene carriers in nature in order to facilitate their removal, and to establish follow-up monitoring programs (Senanan et al. 2007). For highly mobile species, it would be ideal to detect the presence of transgenic individuals before they disperse, since once this initial stage is missed, complete eradication may not be achievable because of the logistics and associated high costs of locating transgenic specimens, particularly in aquatic environments (Baxter et al. 2008). At that point, there may be no other option than to develop acceptable strategies for managing the invaders (Britton et al. 2010).

Contexts similar to these for transgenic organisms have long existed under aquaculture conditions, where genetic interaction between escaped domesticated farmed and local wild fish has occurred (Naylor et al. 
2005, Jonsson and Jonsson 2006, McClure et al. 2008). Attempts to identify hatchery-reared escapees have included external morphology, scale and otolith analysis, biochemical and physiological markers, genetic marking, or scale patterns (Fiske et al. 2005, Glover 2010). The use of genetic markers to identify transgenic specimens would in most cases be a very reliable method, since the transgene is a distinct genetic construct that is either present or absent in the genome. The phenotypic expression of the transgene also may facilitate identification, especially if producing clear external changes such as fluorescent color (Gong et al. 2003). This could be important for the early detection of escaped fish by, for example, fishers that would otherwise not note that the caught specimen is transgenic. However, other types of transgenic fish may not provide such distinctive external markers and may require careful examination if genetic tools are not available or in some circumstances not affordable.

Transgenic fish with elevated growth rate are being developed for commercial purposes, primarily through the introduction of growth hormone $(\mathrm{GH})$ gene constructs. Under culture conditions, these types of transgenic fish grow severalfold faster than their nontransgenic counterparts in early life and can develop altered morphology typically not observed in nontransgenic specimens (Devlin et al. 1995a,b, 2004, Ostenfeld et al. 1998, Nam et al. 2002, 2008). Importantly, however, developmental responses to environmental conditions may alter phenotypic effects in GH transgenic fish (Sundström et al. 2007). This plasticity may complicate the identification of transgenic phenotypes not reared under culture conditions, thereby requiring more sophisticated methods. Typically, transgenic individuals are identified using molecular techniques that require specialized equipment and skilled technical personnel (Michelini et al. 2008, NageswaraRao et al. 2013). Such resources may not be readily available in all locations where transgenic organisms may be expected to be found (Dunham 1999), and consequently other methods may need to be developed.

In the present study, we evaluate the potential of distinguishing GH-transgene-carrying coho salmon (Oncorhynchus kisutch) from non-transgenic conspecifics using either geometric morphometrics (GeoM) analysis or visual examination (VID) by experienced hatchery staff. To take into account the observed plasticity in these fish, we first simulated (within biocontained facilities) the escape of three-month-old hatchery-reared transgenic and non-transgenic fish to an artificial stream and recaptured them after the onset of their smolt migration. Next, we set up a scenario in which the transgenic fish originated from successful spawnings between wild and escaped transgenic specimens. Under this latter scenario, the transgenic fish lived their entire 1.5 years in the artificial stream until seaward migration, when they were captured. In many salmon streams, populations are assessed at this stage through capture in smolt traps, making evaluation of this stage relevant to the context when the detection of a transgenic individual in a salmon population might be feasible.

\section{Methods}

The study was conducted within the transgenic fish facility at the Centre for Aquaculture and Environmental Research (CAER), Fisheries and Oceans, West Vancouver, British Columbia, Canada. This research facility has multiple containment screen systems and is specially designed to prevent the escape of genetically modified fish to the natural environment. Fast-growing genotypes of coho salmon with constitutive expression of growth hormone were initially produced by microinjecting the OnMTGH1 growth hormone gene construct into fertilized eggs from wild parents from the Chehalis River in southwestern British Columbia (Devlin et al. 1994). Stable and characterized strains expressing the transgenic genotype were subsequently developed through crosses with wild fish from the Chehalis River at each generation, and thus contain on average the same genetic background as wild fish, except for the presence of the OnMTGH1 transgene. Details of the production and subsequent performance of the M77 strain used here can be found elsewhere (Devlin et al. 2004). All experiments were conducted under an approved Animal Use Protocol from the Fisheries and Oceans' Pacific Region Animal Care Committee overseeing requirements for the Canadian Council for Animal Care. Informed consent was obtained from all human participants included in the study.

Transgenic fish used in the present experiment were produced by crossing males homozygous for the transgene with wild-caught females from the Chehalis River. Hence, all transgenic fish used in this study were hemizygous for the transgene. Genotypically wild-type fish (i.e., non-transgenic specimens) of the same age were produced by crossing the same wild-caught females with wild-caught males from the same river system. Hence, the hatchery-reared wild-type and transgenic fish were half-sib groups, and the wild-type and transgenic fish reared in the artificial stream were half-sib groups. Because the transgenic fish quickly grow large in the hatchery, we also used a non-transgenic size control where fish were one year older and produced from other wild-caught males and females. It is important to note that the difference in daily specific growth rate in the hatchery between transgenic and wild-type salmon is approximately two- to threefold, which rapidly results in large size differences between genotypes. This difference in growth rate is many times greater than interfamily variation observed among wild-type salmon. All fertilized eggs were incubated in $10^{\circ} \mathrm{C}$ well water until initiation of exogenous feeding.

Transgenic fish simulating first-generation freshwater escapees (Th) were obtained from fish reared under hatchery conditions in $10^{\circ} \mathrm{C}$ well water in a $3.7-\mathrm{m}^{3}$ circular tank and fed commercial salmon feed (Skret- 
TABLE 1. Body measurements of fish studied, where initial is the time when fish escaped to the biocontained artificial stream and final is when fish were photographed and evaluated.

\begin{tabular}{|c|c|c|c|c|c|c|}
\hline \multirow[b]{2}{*}{ Fish type $(n)$} & \multicolumn{2}{|c|}{ Mass (g) } & \multicolumn{2}{|c|}{ Length $(\mathrm{mm})$} & \multicolumn{2}{|c|}{ Condition factor } \\
\hline & Initial & Final & Initial & Final & Initial & Final \\
\hline s. & $\dagger$ & 1 & $\dagger$ & 10 & $\dagger$ & $1.09 \pm$ \\
\hline & $5.5 \pm 0.5$ & 21.8 & $76 \pm 2.2$ & $132 \pm$ & $1.21 \pm 0.01$ & \pm \\
\hline $\mathrm{W}_{1}$ & & $174.1 \pm$ & & $239 \pm 4.7$ & & $1.23 \pm 0.01$ \\
\hline Ts (34) & $\dagger$ & $22.9 \pm 3.0$ & $\dagger$ & $123 \pm 5.8$ & $\dagger$ & $1.03 \pm 0.01$ \\
\hline Th $(8)$ & $34.6 \pm 3.2$ & $126.1 \pm 22.4$ & $145 \pm 4.6$ & $223 \pm 11.8$ & $1.11 \pm 0.02$ & $1.06 \pm 0.04$ \\
\hline
\end{tabular}

Notes: Values are means \pm SE. Numbers in parentheses in fish type denote sample size at final stage. Final size distributions are illustrated in Fig. 2. Cells left blank indicate no data. Fish belonged to five types: transgenic genotypes reared in the hatchery for 3 months and then transferred to an artificial stream for 9 months $(\mathrm{Th})$, transgenic genotypes reared in an artificial stream from the first-feeding stage until 12 months of age (Ts), wild genotype (non-transgenic) reared in the hatchery for 15 months and transferred to large saltwater tanks for 4 months acting as size-control for Th $(\mathrm{Wm})$, wild genotype raised in stream (Ws), and wild genotype raised in hatchery $(\mathrm{Wh})$.

$\dagger$ First-feeding fry that were approximately $0.3 \mathrm{~g}$ and $32 \mathrm{~mm}$.

ting, Vancouver, British Columbia, Canada) to satiation three to five times a day. After three months, 20 individuals were haphazardly sampled from this tank, PIT-tagged (passive integrated transponder) to allow individual identification, and released into a simulated natural stream in August 2005. Mass, length, and condition factor for fish at the time of introduction are given in Table 1. Fish then were reared in the artificial stream until sampling in May 2006 (270 days after release from hatchery), at which point they were recaptured and again measured for length, weighed, and photographed for identification studies. A corresponding wild-type group (Wh) initially reared separately in the hatchery was treated in the same way, but reared together in the stream with the Th group.

Ten days after the first-generation escapes were captured from the stream, 200 wild-type (Ws) and 200 transgenic (Ts) genotypes were transferred at the firstfeeding stage to the artificial stream. Two wild rainbow trout (Oncorhynchus mykiss) predators were introduced in July 2006 and remained in the system until the end of the experiment. One weighed $68 \mathrm{~g}$ and was $180 \mathrm{~mm}$ when released, and had grown to $132 \mathrm{~g}$ and $241 \mathrm{~mm}$ when the experiment ended. The other rainbow trout was $42 \mathrm{~g}$ and measured $160 \mathrm{~mm}$ when introduced, and had grown to $114 \mathrm{~g}$ and $223 \mathrm{~mm}$ when the experiment ended. We were able to capture 69 individuals from the stream in June 2007 (after 376 d). Mass and length were measured before adipose fins were sampled to determine genotype by PCR analysis. Detecting the presence of the transgene with this method has proven reliable across laboratories (Rehbein et al. 2002) and further details are provided elsewhere (Devlin et al. 2004).

A third group of wild-type fish (Wm), used as a size control to the large hatchery-reared transgenic fish, was reared in the hatchery for 18 months before transfer to a saltwater mesocosm tank (12 $\mathrm{m}$ diameter and $3 \mathrm{~m}$ deep circular flow-through tank) in September 2007 and fed with commercial fish feed (Skretting) to satiation. When they had reached a size similar to that of the final size of the hatchery-reared transgenic fish, 24 individuals were captured from the mesocosm and photographed (December 2007).

Condition factor of all fish was calculated as $\mathrm{CF}=10^{6}$ $\times M \times L^{-3}$, where $M$ is mass in grams and $L$ is length in millimeters. Fish were digitally photographed for morphometric analysis and human evaluation. The camera (Sony $\alpha 100$; Sony, Tokyo, Japan) was mounted on a stand and photographs were taken with the same settings for each specimen. However, because fish were not available at the same time, conditions were not identical but made as similar as possible. The left side of each fish was photographed and assessed in all analyses.

\section{Simulated natural stream}

The simulated natural stream (see Plate 1) was constructed with pond liner, and the bottom and most of the sides were covered with large rocks (roughly 5-20 $\mathrm{cm}$ in diameter), with occasional boulders $(30-40 \mathrm{~cm}$ diameter), 1-2-m logs, and a root (Sundström et al. 2010: Fig. 1). It consisted of a $20 \mathrm{~m}$ long and $1.1 \mathrm{~m}$ wide stretch divided by a $0.1 \mathrm{~m}$ thick central wall put in place in a zigzag pattern to within $1.5 \mathrm{~m}$ of the ends, making a looped stream length of $37 \mathrm{~m}$. Water current was created by incoming water (partial flow-through system with natural creek water at $\sim 0.6 \mathrm{~m}^{3} / \mathrm{h}$ ) at one end, and by four pumps (each with a capacity of $\sim 4 \mathrm{~m}^{3} / \mathrm{h}$ ) evenly distributed throughout the stream. The varying distance between the stream sides and the dividing wall and the placement of pumps and boulders created sections with rapid water flow $(2 \mathrm{~m} / \mathrm{s})$ and sections with slow flow $(0.1$ $\mathrm{m} / \mathrm{s})$. Water depth varied between 0.2 and $0.3 \mathrm{~m}$ with three deeper sections (deepest part was $0.4 \mathrm{~m}$ ) at one end. Light followed the natural photoperiod and entered the facility through a semitransparent roof.

Observed but uncontrolled food sources in the system were live natural prey entering the system with the creek water, such as stone fly (Plecoptera) and mayfly (Ephemeroptera) larvae, as well as numerous larvae and adults of mosquitoes (Chironomidae, Culicidae) 


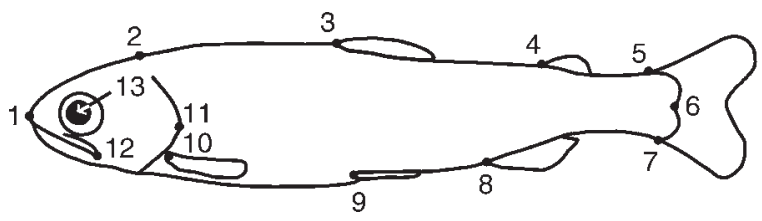

FIG. 1. Locations of the 13 landmarks evaluated with the generalized orthogonal least-squares Procrustes (GPA) procedure to produce relative warp scores used in a canonical discriminant analysis.

and adult spiders (Arachnidae). Supplemental natural feed was provided once a day five days a week, and consisted of frozen water fleas (Dapnia sp.), mysis shrimp (Mysis relicta), and blood worms (Chironomidae), live worms (Tubifex sp. and Lumbricidae), live crickets (Acheta domestica), dried brine shrimp flakes (Artemia spp.), and dried krill (Euphasia spp.). Feeding locations (four to five per feeding) in the stream were chosen haphazardly at each feeding event.

It is important to note that our feeding regimens and the stream at our facility produce non-transgenic fish that are representative of wild fish from nature in terms of average growth history, phenotype, and feeding behavior when assessed under seminatural conditions (Sundström et al. 2007).

\section{Geometric morphometrics}

Genotypic identification through shape analysis using computers was done by means of geometric morphometrics (Adams et al. 2004, Slice 2007). We digitized 13 landmarks from the photographs of the fish (Fig. 1) using the software program tpsDig2 (Rohlf 2008). Variation in shape was small enough to allow statistical analysis to be performed according to tpsSmall (Rohlf 2005). Landmarks were analyzed in tps-Relw, which uses the generalized orthogonal least-squares Procrustes (GPA) procedure to produce both affine (uniform) and non-affine (non-uniform) partial warp scores (Rohlf 2007). From these scores, the first six relative warps were extracted (corresponding to a principal component analysis) and analyzed together with $\log _{10}$ of centroid size in a linear discriminant analysis. We used these relative warps instead of the partial warp scores because only eight individuals were available in one of the groups (Tabachnik and Fidell 2001). We set the prior probability to be equal among groups since our sample is unlikely to accurately reflect any specific real population. Accuracy of the classification was assessed using the leave-one-out cross-validation procedure, where one data point is classified according to the model obtained without that data point, and this procedure was repeated for all data points. Differences in shape between groups were visualized with tps-Relw by looking at the relative warps, which are principal components of the covariance matrix of the partial warp scores and thus summarize as much of the variation in shape as is possible in as few dimensions as possible. Default settings were used in all tps programs.

\section{Human visual identification}

Photographs of all the fish were randomly ordered and assigned a unique number before being assembled into an Adobe pdf file (Adobe, San Jose, California, USA). This file was provided to 11 staff members at CAER, and they were asked to identify each specimen as either of a wild-type or a transgenic genotype. Evaluators were informed that the list of photographs was of wild-type and transgenic individuals, but not how many of each type. They were also asked to provide three main characteristics used to decide whether they considered a fish to be of wild-type or transgenic genotype. Staff members varied in their educational backgrounds (from high school to senior professor), and had 5-20 years of experience in hands-on work with hatchery-reared wildtype and GH-transgenic coho salmon in the laboratory. All staff had experience weighing and measuring fish, and hence had visually observed sides of many fish, although mainly animals that had been reared in the hatchery.

Analysis of the scores had two purposes. First, we looked at whether some of the fish groups were easier than others to distinguish, and second, we looked at whether expert raters were reliable. Fish were given a score of 1 if correctly assigned and 0 if incorrectly assigned. Across raters, if a fish had an average over 0.5 (i.e., at least six of the 11 raters were correct), it was assigned the correct genotype, and if less than 0.5 , it was deemed to have been wrongly classified by the raters.

\section{RESUlts}

Of the 20 transgenic fish that were reared in the hatchery for three months prior to release into the artificial stream, eight were alive at the time of final sampling. The smallest of these fish was $61.3 \mathrm{~g}$ and 189 $\mathrm{mm}$, which is larger than the size at which most normal coho salmon undergo the smolting process (average of typically $<25 \mathrm{~g}$ ) found at this time of year (Völlestad et al. 2004). The largest of the transgenic fish was $238 \mathrm{~g}$ and measured $278 \mathrm{~mm}$. We also obtained 24 size-matched wild-type fish reared in large seawater tanks that ranged between 74.0 and $269 \mathrm{~g}$ and 188 and $278 \mathrm{~mm}$. For the 20 wild-type fish reared in the hatchery and later released, 12 survived, and their sizes ranged between 15.4 and 30.0 $\mathrm{g}$ and measured 119-145 $\mathrm{mm}$. Hence, there was no overlap in size between hatchery-reared wild-type and transgenic fish, so these could have been distinguished based on size alone if captured in a stream environment (Figs. 2 and 3).

Of the 200 first-feeding fry of each genotype released into the simulated natural stream, 63 were recaptured from the artificial stream at the time of smolt migration. Based on PCR analysis, 34 of these fish were transgenic and 29 were wild type (Sundström et al. 2010). In contrast to the hatchery-reared fish, there was great 


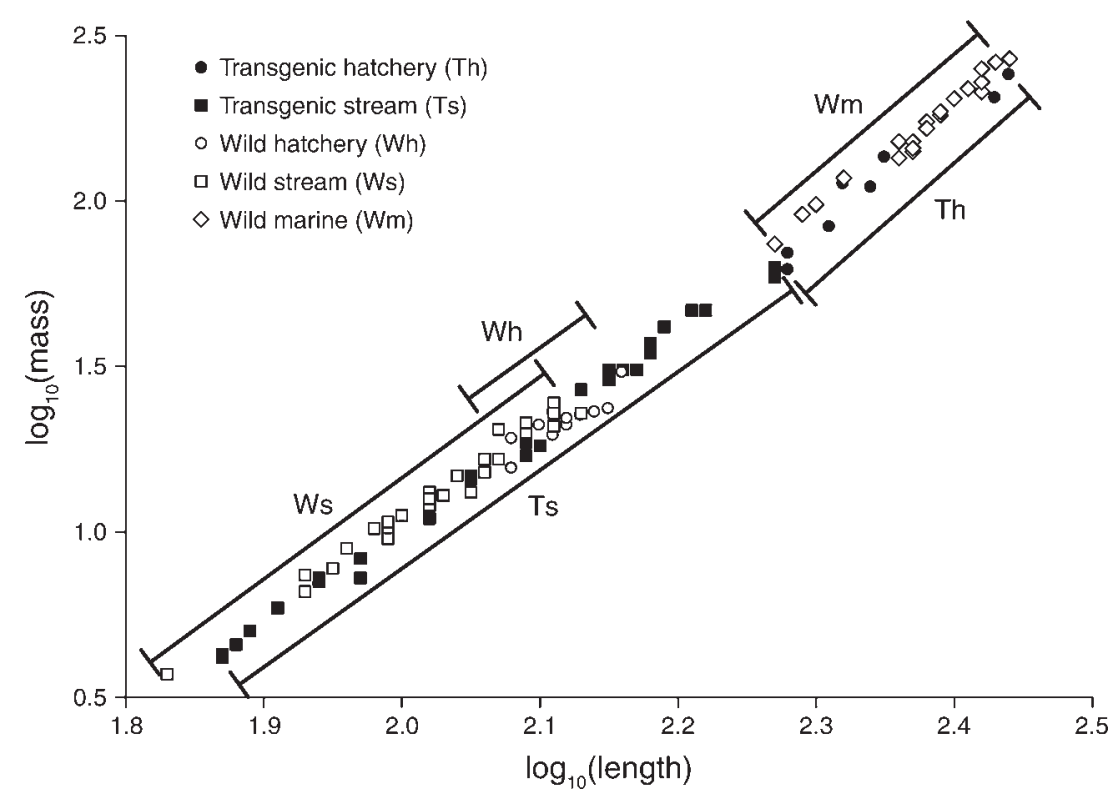

FIG. 2. Length-mass relationship of analyzed fish (length originally measured in mm, mass in g), with data range indicated with lines showing the great overlap in size between wild and transgenic fish reared in an artificial stream and the separation in size between the same genotypes reared in the hatchery. Fish belonged to five groups: transgenic genotypes reared in the hatchery for 3 months and then transferred to an artificial stream for 9 months (Th), transgenic genotypes reared in an artificial stream from the first-feeding stage until 12 months of age (Ts), wild genotype (non-transgenic) reared in the hatchery for 15 months and transferred to large saltwater tanks for 4 months acting as size-control for Th $(\mathrm{Wm})$, wild genotype raised in stream (Ws), and wild genotype raised in hatchery $(\mathrm{Wh})$.

overlap in size between wild-type and transgenic genotypes reared in the stream (Figs. 2 and 3). Wildtype fish ranged from 3.7 to $24.8 \mathrm{~g}$ and 68 to $134 \mathrm{~mm}$, while transgenic fish ranged from 4.2 to $63.3 \mathrm{~g}$ and 74 to $185 \mathrm{~mm}$. It is important to note that even though 12 transgenic fish were larger than any wild type, four of the five smallest fish were transgenic. Wild-type fish reared in the stream were on average smaller than those wild-type fish reared in the hatchery, but there was still overlap, and the hatchery-reared wild-type fish were not larger than the largest of the transgenic genotypes reared in the stream (Fig. 2). Further, the largest transgenic specimen reared in the stream was just as large as the smallest transgenic individual initially reared in the hatchery. These data show that size alone cannot be used as a reliable cue to discriminate between wild-type and transgenic genotypes reared under more nature-like conditions. Against this background, it is relevant to assess the ability of geometric morphometrics and expert evaluation to distinguish transgenic from wild-type genotypes.

\section{Geometric morphometrics}

Geometric morphometry, using the generalized orthogonal least-squares Procrustes (GPA) procedure on landmark data (Fig. 1), produced relative warp scores
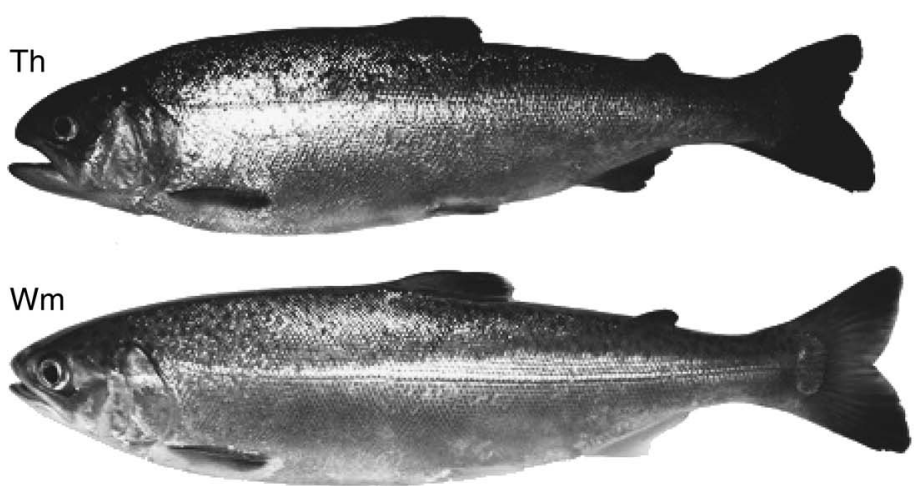

FIG. 3. Representative phenotypes of the five groups of fish (see Fig. 2) scaled to their respective average length.
Ts

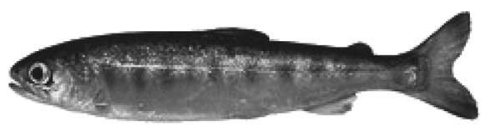

Ws

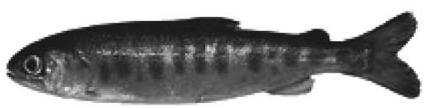

Wh

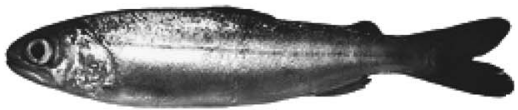

$100 \mathrm{~mm}$ 


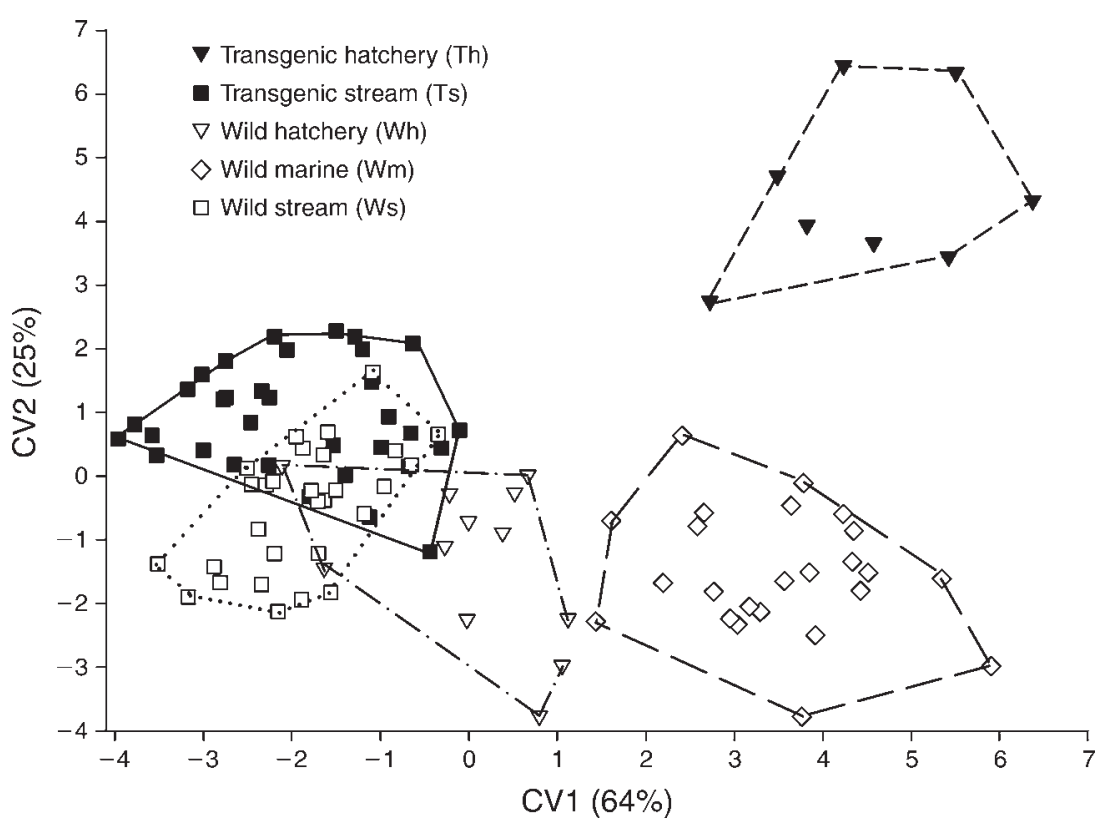

FIG. 4. Classification plot on discriminant functions (canonical variants; CV) 1 (CV1) and 2 (CV2) explaining $91 \%$ of the variation in body shape and display differences in associated variation among five groups of salmon. Numbers in parentheses on each axis indicate the percentage of variation explained by each function. Convex polygons outline each group. See Fig. 2 for details on fish groups.

that discriminated significantly $(\alpha=0.05)$ on the four canonical functions $\left(\chi_{28}^{2}=424, P<0.001\right)$. The first function explained $63.7 \%$ of the variance between the five groups of fish, with the second function explaining $25.3 \%$ (Fig. 4). The third and fourth functions accounted for $6.9 \%$ and $4.1 \%$, respectively, of the remaining variance. The multivariate space distances between groups (a measure of distinctiveness between average phenotypes) were all significant, and were smallest between Wh, Ws, and Ts, and greatest between $\mathrm{Wm}$ and the stream fish (Ws and Ts; Table 2). The leave-one-out classification (Table 3) produced an overall accuracy of $86 \%$. The best assignment was for group Th, with $100 \%$ correct. The least reliable was Ws, with only $72 \%$ correct.

From an ecological risk point of view, the error rate of classifying transgenic specimens as non-transgenic was $7 \%$, with three of the 42 individuals misclassified as a wild type. All of these misclassifications came from transgenic fish reared under naturalized conditions in

TABLE 2. Test of multivariate distances (Mahalanobis distances in between-groups $F$ matrix) between means among groups with $\mathrm{df}=7,96$ and $P<0.001$ for all pairwise comparisons.

\begin{tabular}{lcccc}
\hline \hline Group & Th & Ts & Ws & Wh \\
\hline Ts & 50.0 & & 11.9 & 16.9 \\
Ws & 55.6 & 11.9 & & 10.4 \\
Wh & 36.0 & 16.9 & 10.4 & \\
Wm & 31.7 & 67.2 & 56.6 & 19.2
\end{tabular}

Note: Cells left blank indicate no comparison possible. the artificial stream from the first-feeding stage; one was classified as stream-reared wild-type fish (Ws) and two as a hatchery-reared wild-type fish (Wh). Morphometric data also wrongly classified wild types as transgenic in seven out of 65 cases (11\%). Most problematic was the distinction between the two types of stream-reared fish, with all of the seven errors being between these two groups. Hence, of the 15 misclassifications, eight were between the two genotypes of stream-reared fish. A similar discriminant function analysis, but on only the two stream-reared types (Ts and Ws), where sample size was larger and thus allowed the use of partial warp scores instead of a limited number of relative warps, was no better in separating groups, with five transgenic fish classified as wild type and four wild-type fish classified as transgenic. Hence, this reduced discriminant function

TABLE 3. Leave-one-out classification matrix of wild and transgenic genotypes based on 22 partial warp scores and body length.

\begin{tabular}{lcrrrrr}
\hline \hline & \multicolumn{5}{c}{ Classification } & \\
\cline { 2 - 5 } Actual type & Th & Ts & Ws & Wh & Wm & Correct (\%) \\
\hline Th & $\mathbf{8}$ & & & & & 100 \\
Ts & & $\mathbf{3 1}$ & 1 & 2 & & 91 \\
Ws & & 7 & $\mathbf{2 1}$ & 1 & & 72 \\
Wh & & & 2 & $\mathbf{1 0}$ & & 83 \\
Wm & & & & 2 & $\mathbf{2 2}$ & 92 \\
Total & 8 & 38 & 24 & 15 & 22 & 86 \\
\hline
\end{tabular}

Notes: Numbers denoting correctly assigned fish are shown in boldface type. Cells left blank indicate that no genotypes of the actual type were assigned to this classification (e.g., no Wh genotypes were classified as Ts). 

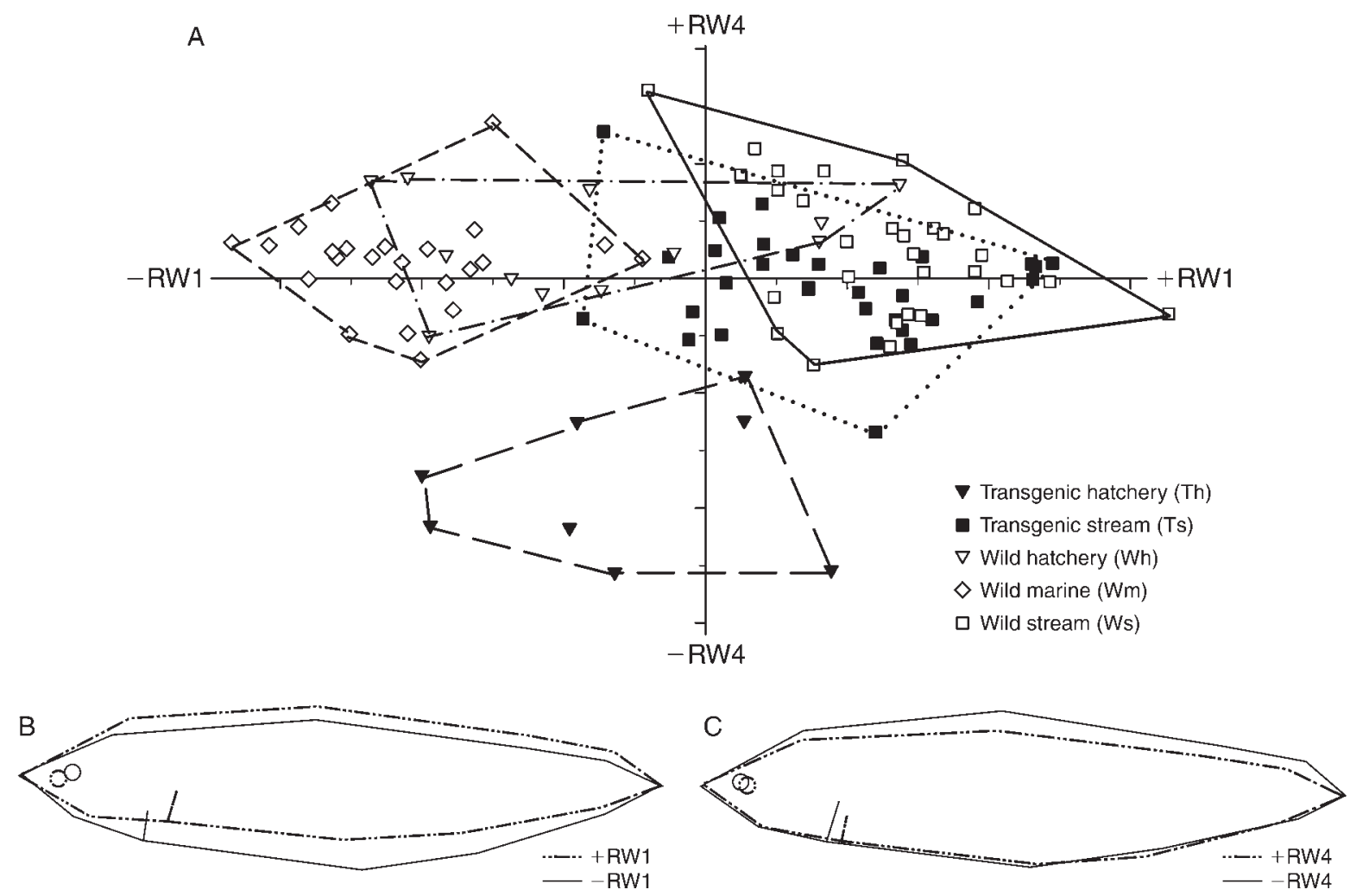

FIG. 5. (A) Relative warps (RW) 1 ( $x$-axis) and 2 ( $y$-axis) explaining $51 \%$ (RW1 $=35 \%$ and RW2 $=7.3 \%$ ) of the deformations observed in the five groups and with corresponding deformation grids for warp extremes. Convex polygons outline each group. (B) Thin-plate spline visualization plot of the extremes of RW1. (C) Thin-plate spline visualization plot of the extremes of RW4. See Fig. 2 for details on fish groups.

analysis improved scoring of the wild-type but reduced accuracy for the transgenic fish.

Although the discriminant analysis used morphometric data to classify most individuals correctly, it did not reveal what features differed among groups. Some idea can be obtained by looking at relative warps (RW) together with visualization of shape deformations using the program tpsRelw (Fig. 5A). Although RW1 and RW2 provided the most explanation for variation in body shape, RW1 and RW4 were chosen in the visualization because they provided the best discrimination between all groups (based on the $F$ statistic from ANOVAs on the first six RW). The extremes of RW1 were associated with slender (positive values) or rotund (negative values) bodies coupled with asymmetric shape changes in the head region. Most distinct were $\mathrm{Wm}$, and to some extent Wh, which clustered on $-\mathrm{RW} 1$, which was associated with fusiform bodies being less symmetrical along the midline and where the eye was further away from the tip of the nose but the head increased in relative size (Fig. 5B). Stream-reared fish clustered more on $+\mathrm{RW} 1$, which was associated with more symmetric body shape along the midline with more similar ventral and dorsal body and relatively smaller heads. - RW4 was associated mainly with a relative increase of dorsal body and slightly smaller head, and this was where $\mathrm{Th}$ clustered (Fig. 5C). This increase in the dorsal part of the body was relatively apparent on the Th fish, where there was a sense of a thickened "neck" (just posterior to the head on the dorsal surface) not obvious in the other phenotypes (Fig. 3).

Because stream-reared fish posed the main problem of discrimination between wild-type and transgenic genotypes (e.g., those that might be derived from spawning events in nature), shape differences between these two fish types were also visualized separately (Fig. 6). A plot between centroid size (basically the same as body length in this case; Pearson correlation between centroid size and body length $R=0.998$ ) and RW1 showed a partial separation between the two genotypes such that wildtype fish generally scored higher on RW1 for a given size (Fig. 6A). However, the relationship was not parallel, and the difference between the two genotypes in the RW1 score decreased with size (analysis of covariance [ANCOVA] interaction between centroid size and genotype; $\left.F_{1,59}=17.5, P<0.001\right)$. As RW1 was associated mainly with the dorsoventral body shape and relative head size, this relationship suggested that smaller wild-type fish have relatively deeper bodies and larger heads than transgenic fish (Fig. 6B), but that this difference disappeared with increasing size. To assess the magnitude of this difference, we measured the distance 

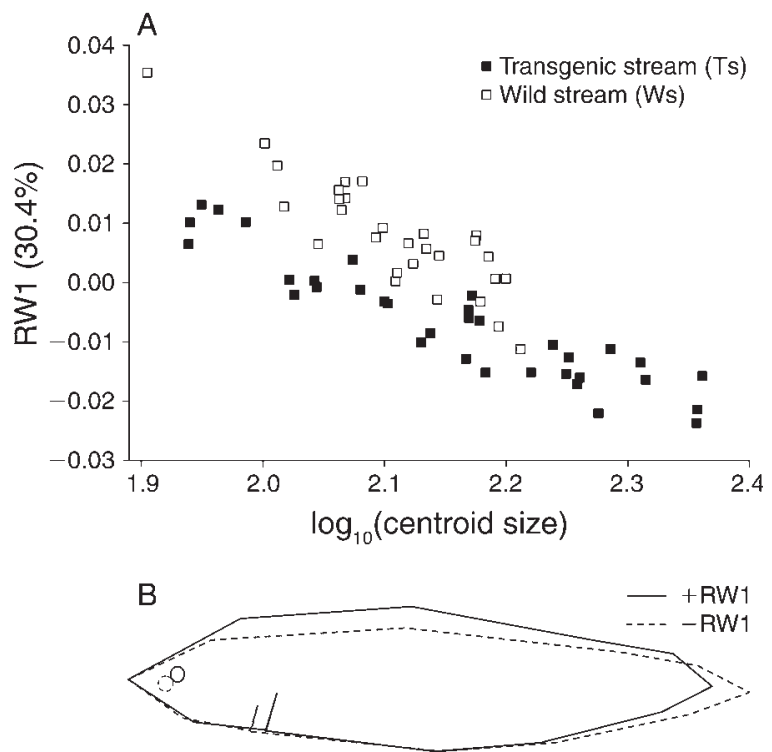

FIG. 6. First relative warp from analysis on Ts and Ws groups only against $\log _{10}$ of centroid size (highly correlated with body length, Pearson's $R=0.998)$. See Fig. 2 for details on fish groups.

between landmarks 3 and 9 (measure of body height; see Fig. 1) and 1 and 11 (measure of head size) and adjusted for body length in an ANCOVA including the interaction between length and genotype. Results of this analysis showed that the difference in distance between landmarks 3 and 9 (body height), comparing streamreared wild-type and transgenic fish, was on average only $0.21 \mathrm{~mm}$ for a fish of $116 \mathrm{~mm}$ (equivalent to a $\log _{10}$ centroid size of 2.15 in Fig. 6A), corresponding to an increase of only $0.8 \%$ in body height in wild-type compared to transgenic fish. The same procedure applied to landmarks 1 and 11 (head size), which showed that this difference between the two genotypes was on average $1.2 \mathrm{~mm}$, corresponding to a $4.9 \%$ increase in relative head size in wild-type fish compared to transgenic. Even though such differences could potentially be reliably measured under field conditions, there was still overlap between the two genotypes, leaving doubt as to the accuracy of this simple method of classification. Further, the difference in slope as detected between centroid size and RW1 suggested that this method may become less reliable as size of the fish increases (Fig. 6).

\section{Human expertise}

The 11 human evaluators scored between 35 and 63 of the 106 fish correctly, with an average of $48.6 \pm 2.6$ (mean $\pm \mathrm{SE})$. Hence, this type of evaluation was not reliable for identifying genotypes, with less than $50 \%$ of the fish correctly assigned. Results for only two raters were different from random, but these raters were correct in less than $50 \%$ of the cases (binomial test, $P$ $<0.015$ for both)
When setting the criteria that $>50 \%$ agreement of the raters would determine genotype, the assignment of individual fish classified only 14 of the 42 transgenic fish as transgenic and 32 of the 65 wild-type fish as of the wild-type genotype. When looking at the different fish types, only transgenic fish initially reared in the hatchery were all correctly classified (Table 4). We note that stream-reared fish were overrepresented as believed to be wild type, whereas the majority of hatchery-reared and large marine tank fish were believed to be transgenic.

The criteria used by the raters to determine genotype can be summarized into three categories. First was the use of relative sizes looking at eye diameter or head size relative to body size, where a relatively small eye/head was considered an indication of transgenic genotype. Second, specific details believed to be associated with transgenic fish included a more blunt nose, cranial deformities, deformed operculum, and reduced pectoral fin. Last, raters relied on general appearance, where transgenic fish were believed to have more rounded bodies (i.e., higher condition), be more likely to have lost scales, and be more silvery and larger than wild type.

\section{DisCUSSION}

Our results indicate that visually identifying a $\mathrm{GH}$ transgenic coho salmon from a wild type with $100 \%$ certainty when found in nature would be difficult, especially so if the former is a second-generation naturalized fish. It is also likely that the younger the first-generation escaped fish are at escape (i.e., having spent less or no time under hatchery conditions), the more difficult they would be to distinguish from wildtype fish, because the transgene has had less time to influence phenotypic development under hatchery conditions (Sundström et al. 2003). We explored both geometric morphometrics and human expert identification and found that whereas the former was somewhat reliable, the latter was no better than chance. To be $100 \%$ certain of the genotype of an unknown specimen would, therefore, require molecular analysis (e.g., Devlin et al. 2004). Despite these limitations, we discuss ways to improve both the analysis of geometric morphometrics and visual identification to become more useful for distinguishing between transgenic and wild-type specimens.

TABLE 4. Classification by expert raters divided by fish group, based on the average of the raters (cut-off for correct assignment $>50 \%$ of reviewers).

\begin{tabular}{lccc}
\hline \hline & \multicolumn{2}{c}{ Naïve raters' genotype } & \\
\cline { 2 - 3 } Group & Transgenic & Wild type & Correct (\%) \\
\hline Th & 8 & 0 & 100 \\
Ts & 6 & 28 & 18 \\
Ws & 4 & 25 & 86 \\
Wh & 9 & 3 & 25 \\
Wm & 19 & 5 & 21 \\
\hline
\end{tabular}



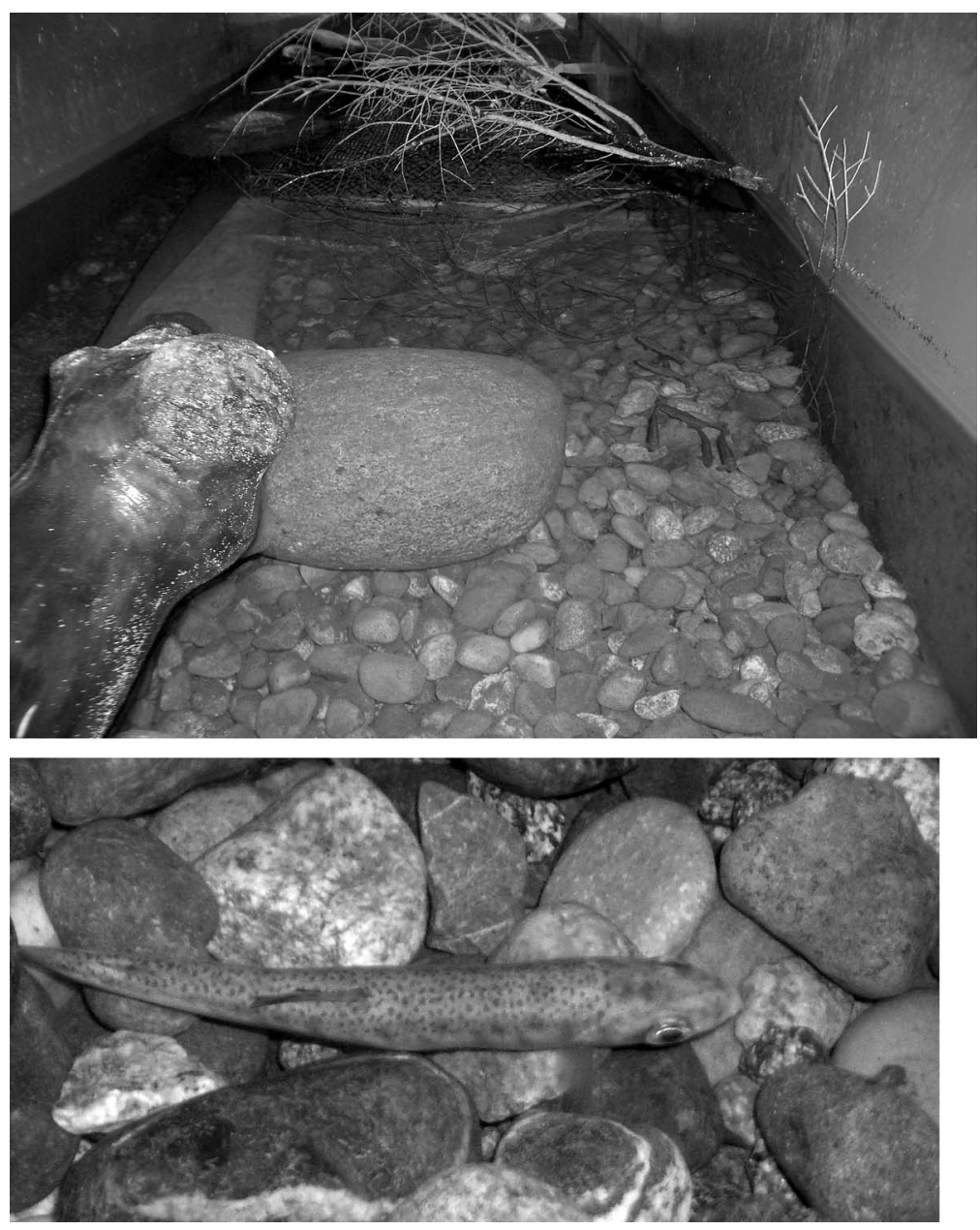

Plate 1. Transgenic and wild-type coho salmon in contained naturalized stream environments. (Above) Complex habitat with a group of shoaling coho salmon; (below) resting coho salmon (genotype undetermined at this stage of the experiment) within an artificial stream. Photo credits: L. F. Sundström, Uppsala University.

Although the poor accuracy of expert identification rates may seem surprising, there are a number of conditions that may explain this and suggest that improvement may be possible. First, although the human experts had seen both genotypes of fish extensively before when reared in hatchery conditions, none of the raters had specific experience in visual identification of stream-reared fish. Hence, much of the erroneous classification arose from preconceived notions of what a transgenic fish looks like (i.e., type Th in Fig. 3). The criteria used and the scoring outcome strongly suggest that the raters used traits easily recognized from transgenic fish reared only in the hatchery. Typically, fast-growing hatchery-reared transgenic fish have smaller eyes and heads, large, bulging abdomens, and a higher condition factor from the larger amount of food consumed compared to wildtype fish (Ostenfeld et al. 1998, Devlin et al. 2012). Such changes in phenotype are highly dependent on indirect effects of enhanced growth rate, since the characteris- tics are much reduced in transgenic animals forced to grow at a wild-type rate by ration restriction (Devlin et al. 2012). These traits had already developed in the Th group after three months in the hatchery and were still easily observable even after 10 months in the stream environment. Thus, it is not surprising that fish belonging to this group had the highest percentage of correctly assigned individuals by both the GeoM and VID methods. Two other criteria used by the raters, body size and silvering, likely explain why the fish derived from large marine tanks (Wm) were largely considered transgenic despite being of wild-type genotype. At the time of migration in early summer, a natural stream would normally not contain coho salmon of the size of Wm fish, and only a transgenic fish from the hatchery would be expected to have acquired such a silvery body. However, sampling in an estuary could potentially catch wild fish of this size together with escaped transgenic fish (Lund 1998 cited in Thorstad et al. 2008). Figs. 3 and 4 and the 
classification based on GeoM (Table 3) clearly demonstrate how group Wm was different from transgenic group Th, and with more experience and specific training, raters would likely be able to tell them apart, and may also show improvement in distinguishing other groups as well.

By far the two most difficult groups to separate were wild-type and transgenic fish reared in the stream environment from the first-feeding stage. Of the 11 fish misclassified by GeoM, eight were between these two groups. Further, the expert raters appeared to (by VID) group both transgenic and non-transgenic stream-reared fish (Ts and Ws) as wild-type fish by default. Fig. 3 illustrates how these two types of fish were also more visually alike than any of the other types examined. The GeoM methods revealed that most of the differences in shape between these two groups were associated with relatively smaller dorsoventral body height and smaller heads in transgenic compared to wild type. As growth hormone can stimulate skeletal growth and lipid metabolism (Johnsson and Björnsson 1994), our data suggest that the similarity of the wild-type and transgenic fish from the stream is not due to an inactivation of the transgene. When transgenic and wild-type fish are fed the same amount of food under hatchery conditions (i.e., pairfed), transgenic fish are longer and leaner for the same mass (reduced condition factor), whereas if these fish are then fed to satiation, they will rapidly outgrow wild type and exhibit increased condition (Raven et al. 2006). However, when fed to satiation, the head of the transgenic fish does not grow isometrically with the body, suggesting that growth hormone affects the growth of the structures of the head differently from those of the rest of the body (Devlin et al. 2012). Thus, it was not unexpected to find that the stream-reared transgenic fish had relatively smaller heads even though they were not necessarily larger than the wild type. A smaller head also developed in hatchery-reared wild-type adult coho salmon compared to adults captured from the wild (Hard et al. 2000), although most of this effect may be due to less-developed secondary sexual characteristics, such as the kype, in the hatchery, which is not relevant for the smolts that we studied.

GeoM was able to detect differences and classify most groups with a reasonably high degree of accuracy, although not $100 \%$. It is possible that GeoM performance could be improved by choosing more or alternative landmarks, since it has been previously noted in other systems that subtleties in morphologies (based on visual observations) can be detected by the geometric shape analysis (Unwin et al. 1999, Solem and Berg 2011). It is conceivable that human observers may be able to learn and discriminate as well or better if they are made aware of which specific traits to seek. GeoM quantifies only shape differences, whereas a human observer can use cues from other aspects such as color and patterns. However, as seen in this study, human performance also may suffer from preconceived notions, and it may be difficult to obtain observers that are trained but without any prejudice.

Although each transgenic line needs to be assessed on a case-by-case basis, the present study reveals an important issue involved in identifying escaped and later-generation transgenic fish in nature. We have shown that even in cases where the transgenic fish may be easily identified when reared under culture conditions, identifying naturalized phenotypes of transgenic fish may be very difficult without resorting to molecular methods even in a species where the phenotypic effects of the transgene is among the greatest observed (Devlin et al. 1994, Nam et al. 2008). The specific strain used in this analysis (M77; Devlin et al. 2004) is one that shows a very high level of growth stimulation but with minimal or no obvious morphological abnormalities (analogous to strains that would presumably be selected for use in production scenarios where they could escape to nature). The results also imply that hatchery phenotypes cannot by default be used as indicators of how easily one could detect the presence of transgenes in nature. Even if there is potential for learning, which may allow human observers to more accurately assign genotypes to naturalized phenotypes, the reliability of identifying the genotypes correctly, at present, appears too low. Over the longer term, and if transgenic fish would be capable of reproducing in the wild, distinguishing between naturalized phenotypes of wild-type and transgenic genotypes may become easier or more difficult, depending on selective forces acting on phenotype (Ahrens and Devlin 2011). In contrast, the high reliability and accuracy of PCR analyses (Rehbein et al. 2002, Devlin et al. 2004) for detecting transgenic animals will not change. Such analyses can be performed within a matter of hours, but do require holding animals and individually identifying them if separation and removal of transgenic from wild-type fish is desired while keeping the latter alive.

In summary, identification of transgenic animals phenotypically should be undertaken in scenarios where they may escape to nature. Further, measures include developing protocols for retrieval of escaped animals, as well as assessing the potential harm that they may cause to ecosystems (Senanan et al. 2007). Because ecological and genetic effects over time may depend on whether or not transgenic individuals can reproduce in nature or not, it may be important to examine specimens reared under natural conditions and not just captivity-reared individuals, and to examine them at reproductively mature stages. Although not at this state currently, GeoM has the potential to be developed into a relatively reliable method of assessing phenotype that, after an initial setup, could work at a relatively low cost. Because GeoM is based on the specific specimens in the analysis, the first step would 
be to create a template database with few but informative landmarks for the relevant species against which any unknown specimen would be compared. This database would need to be developed for a variety of phenotypes, including those of different ages, sizes, and rearing backgrounds. Further, software would have to be developed to facilitate the entry and rapid (i.e., less than 30 seconds) analysis of landmark data from photographs of the examined specimen (i.e., to allow an ID to be generated within a single anesthetization period). Similar identification systems are being developed for a range of species (Kühl and Burghardt 2013), and although they may not provide the same reliability as PCR, they could provide means for large-scale screening of many individuals, which should be coupled with PCR analysis for those individuals where computer analysis generated a transgenic or ambiguous assignment. Further, GeoM analysis has utility with field personnel who are not specifically seeking transgenic animals in sampled populations, but could be made aware of critical diagnostic criteria which could allow detection of transgenic animals in nature that arose from otherwise undetected escape events.

\section{ACKNOWLEDGMENTS}

We are grateful to Malin Andersson, Carlo Biagi, Ben Goh, Jörgen Johnsson, Peter Raven, Dionne Sakhrani, and Steve Tang for their assistance during sampling and/or photography. We also thank the staff that under much agony tried to do their best in genotyping fish from the photographs. Two anonymous reviewers provided insightful comments. The work was carried out with financial support from the Canadian Regulatory System for Biotechnology (R. H. Devlin). L. F. Sundström was funded under a Marie Curie Outgoing International Fellowship under contract MOIF-CT2005-8141 from the European Community's Sixth Framework Programme. The present work does not necessarily reflect the Community's views and in no way anticipates its future policy in this area.

\section{Literature Cited}

Adams, D. C., F. J. Rohlf, and D. E. Slice. 2004. Geometric morphometrics: ten years of progress following the 'Revolution'. Italian Journal of Zoology 71:5-16.

Ahrens, R. N. M., and R. H. Devlin. 2011. Standing genetic variation and compensatory evolution in transgenic organisms: a growth-enhanced salmon simulation. Transgenic Research 20:583-597.

Baxter, P. W. J., J. L. Sabo, C. Wilcox, M. A. McCarthy, and H. P. Possingham. 2008. Cost-effective suppression and eradication of invasive predators. Conservation Biology 22:89-98.

Britton, J. R., R. E. Gozlan, and G. H. Copp. 2010. Managing non-native fish in the environment. Fish and Fisheries 12:256-274.

Devlin, R. H., C. A. Biagi, and T. Y. Yesaki. 2004. Growth, viability and genetic characteristics of GH transgenic coho salmon strains. Aquaculture 236:607-632.

Devlin, R. H., and E. M. Donaldson. 1992. Containment of genetically altered fish with emphasis on salmonids. Pages 229-265 in C. L. Hew and G. L. Fletcher, editors. Transgenic fish. World Scientific Publishers, Singapore.

Devlin, R. H., D. Sakhrani, C. A. Biagi, and K. W. Eom. 2010. Occurrence of incomplete paternal-chromosome retention in
GH-transgenic coho salmon being assessed for reproductive containment by pressure-shock-induced triploidy. Aquaculture 304:66-78.

Devlin, R. H., L. F. Sundström, and W. M. Muir. 2006. Interface of biotechnology and ecology for environmental risk assessments of transgenic fish. Trends in Biotechnology 24:89-97.

Devlin, R. H., W. E. Vandersteen, M. Uh, and E. D. Stevens. 2012. Genetically modified growth affects allometry of eye and brain in salmonids. Canadian Journal of Zoology 90:193-202.

Devlin, R. H., T. Y. Yesaki, C. A. Biagi, E. M. Donaldson, P. Swanson, and W. K. Chan. 1994. Extraordinary salmon growth. Nature 371:209-210.

Devlin, R. H., T. Y. Yesaki, E. M. Donaldson, S. J. Du, and C. L. Hew. 1995a. Production of germline transgenic Pacific salmonids with dramatically increased growth-performance. Canadian Journal of Fisheries and Aquatic Sciences 52:1376-1384.

Devlin, R. H., T. Y. Yesaki, E. M. Donaldson, and C. L. Hew. 1995b. Transmission and phenotypic effects of an antifreeze GH gene construct in coho salmon (Oncorhynchus kisutch). Aquaculture 137:161-169.

Dunham, R. A. 1999. Utilization of transgenic fish in developing countries: potential benefits and risks. Journal of the World Aquaculture Society 30:1-11.

Fiske, P., R. A. Lund, and L. P. Hansen. 2005. Identifying fish farm escapees. Pages 659-680 in S. X. Cadrin, K. D. Friedland, and J. D. Waldman, editors. Stock identification methods. Elsevier Academic Press, Amsterdam, Netherlands.

Glover, K. A. 2010. Forensic identification of fish farm escapees: the Norwegian experience. Aquaculture Environment Interactions 1:1-10.

Gong, Z. Y., H. Y. Wan, T. L. Tay, H. Wang, M. R. Chen, and T. Yan. 2003. Development of transgenic fish for ornamental and bioreactor by strong expression of fluorescent proteins in the skeletal muscle. Biochemical and Biophysical Research Communications 308:58-63.

Hard, J. J., B. A. Berejikian, E. P. Tezak, S. L. Schroder, C. M. Knudsen, and L. T. Parker. 2000. Evidence for morphometric differentiation of wild and captively reared adult coho salmon: a geometric analysis. Environmental Biology of Fishes 58:61-73.

Johnsson, J. I., and B. T. Björnsson. 1994. Growth-hormone increases growth rate, appetite and dominance in juvenile rainbow trout, Oncorhynchus mykiss. Animal Behaviour 48:177-186.

Jonsson, B., and N. Jonsson. 2006. Cultured Atlantic salmon in nature: a review of their ecology and interaction with wild fish. ICES Journal of Marine Science 63:1162-1181.

Kapuscinski, A. R., K. R. Hayes, S. Li, and G. Dana, editors. 2007. Environmental risk assessment of genetically modified organisms. Volume 3. Methodologies for transgenic fish. CAB International, Wallingford, UK.

Kühl, H. S., and T. Burghardt. 2013. Animal biometrics: quantifying and detecting phenotypic appearance. Trends in Ecology \& Evolution 28:432-441.

Lovell, S., S. Stone, and L. Fernandez. 2006. The economic impacts of aquatic invasive species: a review of the literature. Agricultural and Resource Economics Review 35:195-208.

Lund, R. A. 1998. Rømt oppdrettslaks i Namsen og nære sjøområder. Fiske etter rømt oppdrettsfisk i elveutløpet. NINA Oppdragsmelding 564:1-14.

Mair, G. C., Y. K. Nam, and I. I. Solar. 2007. Risk management: reducing risk through confinement of transgenic fish. Pages 209-238 in A. R. Kapuscinski, K. R. Hayes, S. Li, and G. Dana, editors. Environmental risk assessment of genetically modified organisms. Volume 3 . Methodologies for transgenic fish. CAB International, Wallingford, UK.

McClure, M. M., F. M. Utter, C. Baldwin, R. W. Carmichael, P. F. Hassemer, P. J. Howell, P. Spruell, T. D. Cooney, H. A. 
Schaller, and C. E. Petrosky. 2008. Evolutionary effects of alternative artificial propagation programs: implications for viability of endangered anadromous salmonids. Evolutionary Applications 1:356-375.

Michelini, E., P. Simoni, L. Cevenini, L. Mezzanotte, and A. Roda. 2008. New trends in bioanalytical tools for the detection of genetically modified organisms: an update. Analytical and Bioanalytical Chemistry 392:355-367.

Nageswara-Rao, M., C. Kwit, S. Agarwal, M. Patton, J. Skeen, J. Yuan, R. Manshardt, and C. Stewart. 2013. Sensitivity of a real-time PCR method for the detection of transgenes in a mixture of transgenic and non-transgenic seeds of papaya (Carica papaya L.). BMC Biotechnology 13:69.

Nam, Y. K., Y. S. Cho, H. J. Cho, and D. S. Kim. 2002. Accelerated growth performance and stable germ-line transmission in androgenetically derived homozygous transgenic mud loach, Misgurnus mizolepis. Aquaculture 209:257-270.

Nam, Y. K., N. Maclean, G. Hwang, and D. S. Kim. 2008. Autotransgenic and allotransgenic manipulation of growth traits in fish for aquaculture: a review. Journal of Fish Biology 72:1-26.

Naylor, R., K. Hindar, I. A. Fleming, R. Goldburg, S. Williams, J. Volpe, F. Whoriskey, J. Eagle, D. Kelso, and M. Mangel. 2005. Fugitive salmon: assessing the risks of escaped fish from net-pen aquaculture. BioScience 55:427437.

Ostenfeld, T. H., E. McLean, and R. H. Devlin. 1998. Transgenesis changes body and head shape in Pacific salmon. Journal of Fish Biology 52:850-854.

Raven, P. A., R. H. Devlin, and D. A. Higgs. 2006. Influence of dietary digestible energy content on growth, protein and energy utilization and body composition of growth hormone transgenic and non-transgenic coho salmon (Oncorhynchus kisutch). Aquaculture 254:730-747.

Reaser, J., L. Meyerson, Q. Cronk, M. De Poorter, L. Eldrege, E. Green, M. Kairo, P. Latasi, R. Mack, and J. Mauremootoo. 2007. Ecological and socioeconomic impacts of invasive alien species in island ecosystems. Environmental Conservation 34:98-111.

Rehbein, H., R. H. Devlin, and H. Rueggeberg. 2002. Detection of a genetic alteration and species identification of coho salmon (Oncorhynchus kisutch): a collaborative study. European Food Research and Technology 214:352-355.

Rohlf, F. J. 2005. tpsSmall, version 1.15. Department of Ecology and Evolution, State University of New York, Stony Brook, New York, USA

Rohlf, F. J. 2007. tpsRelw, relative warp analysis, version 1.47. Department of Ecology and Evolution, State University of New York, Stony Brook, New York, USA.

Rohlf, F. J. 2008. tpsDig, digitize landmarks and outlines, version 2.12. Department of Ecology and Evolution, State University of New York, Stony Brook, New York, USA.
Senanan, W., J. J. Hard, A. Alcivar-Warren, J. Trisak, M. Zakaria-Ismail, and M. Lorenzo Hernandez. 2007. Risk management: post-approval monitoring and remediation. Pages 239-271 in A. R. Kapuscinski, K. R. Hayes, S. Li, and G. Dana, editors. Environmental risk assessment of genetically modified organisms. Volume 3. Methodologies for transgenic fish. CAB International, Wallingford, UK.

Slice, D. E. 2007. Geometric morphometrics. Annual Review of Anthropology 36:261-281.

Snow, A. A., D. A. Andow, P. Gepts, E. M. Hallerman, A. Power, J. M. Tiedje, and L. L. Wolfenbarger. 2005. Genetically engineered organisms and the environment: current status and recommendations. Ecological Applications 15:377-404.

Solem, Ø., and O. K. Berg. 2011. Morphological differences in parr of Atlantic salmon Salmo salar from three regions in Norway. Journal of Fish Biology 78:1451-1469.

Sundström, L. F., R. H. Devlin, J. I. Johnsson, and C. A. Biagi. 2003. Vertical position reflects increased feeding motivation in growth hormone transgenic coho salmon (Oncorhynchus kisutch). Ethology 109:701-712.

Sundström, L. F., M. Lõhmus, and R. H. Devlin. 2010. Migration and growth potential of coho salmon smolts: implications for ecological impacts from growth-enhanced fish. Ecological Applications 20:1372-1383.

Sundström, L. F., M. Lõhmus, W. E. Tymchuk, and R. H. Devlin. 2007. Gene-environment interactions influence ecological consequences of transgenic animals. Proceedings of the National Academy of Sciences USA 104:3889-3894.

Tabachnik, B. G., and L. S. Fidell. 2001. Using multivariate statistics. Fourth edition. Allyn \& Bacon, Needham Heights, Massachusetts, USA.

Thorstad, E., I. Fleming, P. McGinnity, D. Soto, V. Wennevik, and F. Whoriskey. 2008. Incidence and impacts of escaped farmed Atlantic salmon Salmo salar in nature. NINA Special Report 36. Norwegian Institute for Nature Research, Tungasletta, Norway.

Tiedje, J. M., R. K. Colwell, Y. L. Grossman, R. E. Hodson, R. E. Lenski, R. N. Mack, and P. J. Regal. 1989. The planned introduction of genetically engineered organisms: ecological considerations and recommendations. Ecology 70:298-315.

Unwin, M., M. Kinnison, and T. Quinn. 1999. Exceptions to semelparity: postmaturation survival, morphology, and energetics of male chinook salmon (Oncorhynchus tshawytscha). Canadian Journal of Fisheries and Aquatic Sciences 56:1172-1181.

Völlestad, L. A., J. Peterson, and T. P. Quinn. 2004. Effects of freshwater and marine growth rates on early maturity in male coho and chinook salmon. Transactions of the American Fisheries Society 133:495-503.

\section{Supplemental Material}

\section{Data Availability}

Data associated with this paper are available from Uppsala University: http://urn.kb.se/resolve?urn=urn:nbn:se:uu:diva-242002 